\title{
New Stability Criterion for Discrete-Time Genetic Regulatory Networks with Time-Varying Delays and Stochastic Disturbances
}

\author{
Yanfeng Zhao, ${ }^{1,2}$ Jihong Shen, ${ }^{3}$ and Dongyan Chen ${ }^{4}$ \\ ${ }^{1}$ College of Automation, Harbin Engineering University, Harbin 150001, China \\ ${ }^{2}$ Graduate Department, Harbin University of Science and Technology, Harbin 150080, China \\ ${ }^{3}$ College of Science, Harbin Engineering University, Harbin 150001, China \\ ${ }^{4}$ Department of Applied Mathematics, Harbin University of Science and Technology, Harbin 150080, China \\ Correspondence should be addressed to Yanfeng Zhao; zhaoyanfeng@hrbust.edu.cn
}

Received 9 February 2016; Revised 5 May 2016; Accepted 12 May 2016

Academic Editor: Jose J. Muñoz

Copyright (c) 2016 Yanfeng Zhao et al. This is an open access article distributed under the Creative Commons Attribution License, which permits unrestricted use, distribution, and reproduction in any medium, provided the original work is properly cited.

We propose an improved stability condition for a class of discrete-time genetic regulatory networks (GRNs) with interval timevarying delays and stochastic disturbances. By choosing an augmented novel Lyapunov-Krasovskii functional which contains some triple summation terms, a less conservative sufficient condition is obtained in terms of linear matrix inequalities (LMIs) by using the combination of the lower bound lemma, the discrete-time Jensen inequality, and the free-weighting matrix method. It is shown that the proposed results can be readily solved by using the Matlab software. Finally, two numerical examples are provided to illustrate the effectiveness and advantages of the theoretical results.

\section{Introduction}

Genetic regulatory networks (GRNs), which are structured by the networks of the regulatory interactions among DNA, RNA, and proteins, have become an important research domain in the biological and biomedical sciences. Basically, there are two basic types of the genetic regulatory network models including the Boolean model [1] and the differential/difference equation model [2]. It should be noted that it is difficult to give an accurate mathematical model for the GRNs due to various reasons such as the limitations on the experimental data, the noise in the data extraction, and enormous complexity of the actual biological systems. So far, a lot of effective analysis methods have been developed in the relevant literatures, such as [3-5] and the references therein. Accordingly, the stability analysis issues for GRNs have drawn a great deal of attention and many important results have been given in this area based on the Lyapunov stability theory; see, for example, $[6,7]$.

It is revealed that the time delay is inevitable in the GRNs due to the slow biochemical reactions including the actual regulation, transcription, translation, diffusion, and translocation, especially in that of a eukaryotic cell [8]. The existence of the time delays would degrade the performance of the dynamical systems and even lead to the instability [9-16]. Hence, it is not surprising that there are a great number of results concerning the stability analysis for GRNs with time delays. For example, a functional differential equation model has been introduced in [9] for GRNs with time delays and its local stability and bifurcation have been discussed. In [11], the discrete-time analog of the continuous-time GRNs has been formulated and a stability analysis condition has been given for the discrete-time GRNs with time delays. It is worth noting that the above delayed GRNs are all concerning the deterministic delays. However, in many practical systems (e.g., networked control systems), the network-induced delay often appears as the time-varying feature. In [17], the robust stability problem has been addressed for GRNs with time-varying delays. Subsequently, the improved stability criteria have been established for GRNs with interval timevarying delays [18] and infinite-distributed delays [19]. In [8], the authors have studied a class of GRNs with same 
transcriptional delays and translation delays which take values in a finite set governed by a Markov chain with known transition probabilities.

Note that the models in $[6,9]$ are all described by the differential equations. However, during implementing a continuous-time genetic regulatory network (GRN) for computer simulation and experimental or computational purposes, it is common to discretize the continuous-time GRN to a discrete-time analogue. Therefore, there is a need to study the dynamics behaviors of the discrete-time GRNs. Up to now, there is a little work on the discrete-time GRNs with delays [20-23]. Recently, the asymptotic stability problem has been investigated in [24] for a class of discrete-time uncertain stochastic GRNs with time-varying delays, where a set of sufficient conditions to ensure the stability has been derived in terms of LMIs, while the exponential stability analysis and the robust $H_{\infty}$ control problems for discrete-time GRNs have been investigated in $[25,26]$. On the other hand, it is well recognized that the noise, which involves the intrinsic fluctuation and extrinsic noise, may impose great effect on system performance of the complex systems [27-31]. For example, the global robust power-rate stability problem has been studied in [28] for uncertain GRNs with unbounded time-varying delay and noise perturbations based on the Lyapunov method, Itô's differential formula, and LMI technique. In [30], the authors have investigated the stability problem for GRNs with time-varying delays and stochastic fluctuations, where some sufficient conditions have been given to guarantee the asymptotic stability and exponential stability of the addressed GRNs. So far, it is worth mentioning that there is a great need to analyze the stability problem of delayed GRNs with stochastic disturbances by proposing new analysis method with less conservatism, which has wide applications in the biological/biomedical sciences; see, for example, [32, 33].

In this paper, we aim to obtain an improved stability criterion for discrete-time GRNs with interval time-varying delays and stochastic disturbances based on the existing methods. A new augmented Lyapunov-Krasovskii functional is firstly constructed which contains some triple summation terms. Furthermore, both the discrete-time Jensen inequality and the lower bound lemma are adopted to handle the summation terms. As in [34], it is easy to see that the lower bound theorem can achieve better results than those based on the Jensen inequality for the stability analysis of the delayed systems. Therefore, we revisit the stability analysis problem for GRNs with time-varying delays and the stochastic disturbances by fully taking the lower bound lemma, the discretetime Jensen inequality, and the free-weighting matrix method into account and propose an improved stability criterion with less conservatism compared with the results in $[20,21]$. The main contributions of this paper are highlighted as follows: (1) both the discrete-time Jensen inequality and the well-known reciprocally convex combination approach are adopted to handle the triple summation terms; hence more information of the time delays is reflected in the main results; and (2) a new sufficient condition with less conservatism is given for the considered discrete delayed GRNs in terms of the LMI method. Finally, two numerical examples are included to show the effectiveness of the proposed method.

Notations. For any matrix $A, A>0$ means that $A$ is symmetric positive definite. $E[\cdot]$ stands for the mathematical expectation operator. The superscript $T$ represents the transpose of the matrix. I represents the identity matrix with appropriate dimensions. $(\mho, \mathscr{F}, \mathbb{P})$ is a probability space, $\mho$ is the sample space, $\mathscr{F}$ is the $\sigma$-algebra of subsets of the sample space, and $\mathbb{P}$ is the probability measure on $\mathscr{F}$. For simplicity, in symmetric block matrices, we often use $*$ to represent the term that is induced by symmetry.

\section{Model Description and Preliminaries}

Consider the following discrete-time GRNs with timevarying delays, $n$ mRNAs, and $n$ proteins which are described by the difference equations:

$$
\begin{aligned}
M_{i}(k+1)= & e^{-a_{i} h} M_{i}(k) \\
& +\phi_{i}(h)\left[\sum_{j=1}^{n} b_{i j} f_{j}\left(P_{j}(k-\tau(k))\right)+W_{i}\right], \\
P_{i}(k+1)= & e^{-c_{i} h} P_{i}(k)+\varphi_{i}(h)\left[d_{i} M_{i}(k-d(k))\right],
\end{aligned}
$$

where $M_{i}(k) \in \mathbb{R}$ and $P_{i}(k) \in \mathbb{R}(i=1,2, \ldots, n)$ are the concentrations of mRNA and protein of the $i$ th node, respectively. $h$ is a positive real number denoting a uniform discretionary step size, and $a_{i}>0$ and $c_{i}>0$ are the degradation rates of mRNA and protein, respectively. $d_{i}$ is the translation rate, and $\tau(k)>0$ and $d(k)>0$ are the timevarying delays denoting the feedback regulation delay and the translation delay satisfying $0<\tau_{m} \leq \tau(k) \leq \tau_{M}$ and $0<$ $d_{m} \leq d(k) \leq d_{M}$. Let $W_{i}=\sum_{j \in \mathcal{N}_{i}} v_{i j}$, where $v_{i j}$ is a bounded constant and denotes the dimensionless transcriptional rate of transcription factor $j$ to $i$, and $\mathcal{N}_{i}$ is the set of all $j$ which is a repressor of gene $i$. Moreover, $\phi_{i}(h)=\left(1-e^{-a_{i} h}\right) / a_{i}$ and $\varphi_{i}(h)=\left(1-e^{-c_{i} h}\right) / c_{i}$. Obviously, we can see that $\phi_{i}(h)>0$ and $\varphi_{i}(h)>0$ hold. The coupling coefficients $b_{i j}(i, j=1,2, \ldots, n)$ are defined as follows:

$$
\begin{aligned}
& b_{i j} \\
& = \begin{cases}v_{i j} & \text { if transcription factor } j \text { is an activator of gene } i, \\
0 & \text { if there is no link from node } j \text { to } i, \\
-v_{i j} & \text { if transcription factor } j \text { is an repressor of gene } i .\end{cases}
\end{aligned}
$$

In addition, the nonlinear function $f_{j}(\cdot) \in \mathbb{R}$ represents the feedback regulation of the protein on the transcription. It is a monotonic function in the Hill form; that is, $f_{j}(x)=$ $\left(x / \beta_{j}\right)^{H_{j}} /\left(1+\left(x / \beta_{j}\right)^{H_{j}}\right)$, where $H_{j}$ is the Hill coefficient and $\beta_{j}$ is a positive constant.

Next, rewrite system (1) as the following compact form:

$$
\begin{aligned}
& M(k+1)=A M(k)+B f(P(k-\tau(k)))+V, \\
& P(k+1)=C P(k)+D M(k-d(k)),
\end{aligned}
$$


where

$$
\begin{aligned}
& M(k)=\left[M_{1}(k), M_{2}(k), \ldots, M_{n}(k)\right]^{T}, \\
& P(k)=\left[P_{1}(k), P_{2}(k), \ldots, P_{n}(k)\right]^{T}, \\
& V=\left[\phi_{1}(h) W_{1}, \phi_{2}(h) W_{2}, \ldots, \phi_{n}(h) W_{n}\right]^{T}, \\
& f(P(k-\tau(k)))=\left[f_{1}\left(P_{1}(k-\tau(k))\right),\right. \\
& \left.f_{2}\left(P_{2}(k-\tau(k))\right), \ldots, f_{n}\left(P_{n}(k-\tau(k))\right)\right]^{T}, \\
& A=\operatorname{diag}\left\{e^{-a_{1} h}, e^{-a_{2} h}, \ldots, e^{-a_{n} h}\right\} \text {, } \\
& C=\operatorname{diag}\left\{e^{-c_{1} h}, e^{-c_{2} h}, \ldots, e^{-c_{n} h}\right\}, \\
& D=\operatorname{diag}\left\{\varphi_{1}(h) d_{1}, \varphi_{2}(h) d_{2}, \ldots, \varphi_{n}(h) d_{n}\right\}, \\
& B=\operatorname{diag}\left(\begin{array}{cccc}
\phi_{1}(h) b_{11} & \phi_{1}(h) b_{12} & \cdots & \phi_{1}(h) b_{1 n} \\
\phi_{2}(h) b_{21} & \phi_{2}(h) b_{22} & \cdots & \phi_{2}(h) b_{2 n} \\
\vdots & \vdots & \vdots & \vdots \\
\phi_{n}(h) b_{n 1} & \phi_{n}(h) b_{n 2} & \cdots & \phi_{n}(h) b_{n n}
\end{array}\right) .
\end{aligned}
$$

Let $\left[M^{*^{T}}, P^{*^{T}}\right]=\left[M_{1}^{*}, M_{2}^{*}, \ldots, M_{n}^{*}, P_{1}^{*}, P_{2}^{*}, \ldots, P_{n}^{*}\right]$ be an equilibrium point of system (3). Then, it follows that

$$
\begin{aligned}
M^{*} & =A M^{*}+B f\left(P^{*}\right)+V, \\
P^{*} & =C P^{*}+D M^{*} .
\end{aligned}
$$

Subsequently, we shift the equilibrium point $\left[M^{*^{T}}, P^{*^{T}}\right]$ of system (3) to the origin point through the transformations $x(k)=M(k)-M^{*}, y(k)=P(k)-P^{*}$. Then, system (3) can be transformed into the following form:

$$
\begin{aligned}
& x(k+1)=A x(k)+B g(y(k-\tau(k))), \\
& y(k+1)=C y(k)+D x(k-d(k)),
\end{aligned}
$$

where $g(y(k))=f\left(y(k)+P^{*}\right)-f\left(P^{*}\right)$.

When the stochastic perturbations are taken into account, system (6) becomes

$$
\begin{aligned}
& x(k+1)=A x(k)+B g(y(k-\tau(k))) \\
& \quad+\sigma[x(k), x(k-d(k)), y(k), y(k-\tau(k))] \omega(k), \\
& y(k+1)=C y(k)+D x(k-d(k)),
\end{aligned}
$$

where $\omega(k)$ is a one-dimensional Gaussian white noise sequence on a probability space $(\mho, \mathscr{F}, \mathbb{P})$ with

$$
\begin{aligned}
\mathbb{E}[\omega(k)] & =0, \\
\mathbb{E}\left[\omega^{2}(k)\right] & =1, \\
\mathbb{E}[\omega(i) \omega(j)] & =0 \quad(i \neq j) .
\end{aligned}
$$

The noise intensity matrix $\sigma[x(k), x(k-d(k)), y(k), y(k-$ $\tau(k))]$ satisfies

$$
\begin{aligned}
\sigma^{T} & {[x(k), x(k-d(k)), y(k), y(k-\tau(k))] \omega(k) } \\
\cdot & \sigma[x(k), x(k-d(k)), y(k), y(k-\tau(k))] \omega(k) \\
& \leq x^{T}(k) H_{1} x(k)+x^{T}(k-d(k)) H_{2} x(k-d(k)) \\
& +y^{T}(k) H_{3} y(k)+y^{T}(k-d(k)) H_{4} y(k-d(k)),
\end{aligned}
$$

where $H_{1}, H_{2}, H_{3}$, and $H_{4}$ are known constant matrices with appropriate dimensions.

Assumption 1. For $i=1,2, \ldots, n$ and any $s_{1}, s_{2} \in \mathbb{R}$ with $s_{1} \neq$ $s_{2}, g_{i}(\cdot)$ is a monotonically increasing function and satisfies that

$$
0 \leq \frac{g_{i}\left(s_{1}\right)-g_{i}\left(s_{2}\right)}{s_{1}-s_{2}} \leq l_{i}, \quad g_{i}(0)=0,
$$

where $l_{i}$ are known constants.

Remark 2. Assumption 1 has been commonly used in the literature, for example, [11]. In fact, it is easy to check that the left-hand side of (10) holds as long as $g_{i}(\cdot)$ is a monotonically increasing function.

To end this section, we introduce the following lemmas which will be frequently used in the derivation of the main results.

Lemma 3 (see [26] (discrete-time Jensen inequality)). For any $Z>0$, two scalars $\tau_{m}$ and $\tau_{M}$ with $\tau_{m} \leq \tau_{M}$, and any vector-valued function $\eta(i)$, it follows that

$$
\begin{aligned}
& -\sum_{i=k-\tau_{M}}^{k-\tau_{m}-1} \eta^{T}(i) Z \eta(i) \leq \frac{-1}{\left(\tau_{M}-\tau_{m}\right)}\left(\sum_{i=k-\tau_{M}}^{k-\tau_{m}-1} \eta^{T}(i)\right) \\
& \cdot Z\left(\sum_{i=k-\tau_{M}}^{k-\tau_{m}-1} \eta(i)\right), \\
& -\sum_{j=-\tau_{M}}^{-\tau_{m}-1} \sum_{i=k+j}^{k-1} \eta^{T}(i) Z \eta(i) \\
& \leq \frac{-2}{\left(\tau_{M}-\tau_{m}\right)\left(\tau_{M}+\tau_{m}+1\right)}\left(\sum_{j=-\tau_{M}}^{-\tau_{m}-1} \sum_{i=k+j}^{k-1} \eta^{T}(i)\right) \\
& \cdot Z\left(\sum_{j=-\tau_{M}}^{-\tau_{m}-1} \sum_{i=k+j}^{k-1} \eta(i)\right) .
\end{aligned}
$$

Lemma 4 (see [34] (lower bounds theorem)). Let $f_{1}, f_{2}$, $\ldots, f_{N}: \mathbb{R}^{m} \mapsto \mathbb{R}$ have positive values in an open subset $D$ of $\mathbb{R}^{m}$. Then, the reciprocally convex combination of $f_{i}$ over $D$ satisfies

$$
\min _{\left\{\alpha_{i} \mid \alpha_{i}>0, \sum_{i} \alpha_{i}=1\right\}} \sum_{i} \frac{1}{\alpha_{i}} f_{i}(t)=\sum_{i} f_{i}(t)+\max _{g_{i, j}(t)} \sum_{i \neq j} g_{i, j}(t)
$$


with

$$
\begin{aligned}
& \left\{g_{i, j}: \mathbb{R}^{m} \longmapsto \mathbb{R}, g_{j, i}(t) \triangleq g_{i, j}(t),\left[\begin{array}{cc}
f_{i}(t) & g_{i, j}(t) \\
g_{i, j}(t) & f_{j}(t)
\end{array}\right]\right. \\
& \quad \geq 0\} .
\end{aligned}
$$

\section{Main Results}

In this section, a new Lyapunov-Krasovskii functional is firstly constructed by taking some triple summation terms into account. Subsequently, we give a new stability condition for the addressed GRNs with time-varying delays and stochastic disturbances by using the combination of the lower bound lemma, the discrete-time Jensen inequality, and the free-weighting matrices method. It is worthwhile to mention that the presented sufficient condition can be easily checked by using the Matlab LMI toolbox.

Theorem 5. For given positive scalars $d_{m}, d_{M}, \tau_{m}$, and $\tau_{M}$, if there exist scalar $\rho>0$, matrices $P_{1}>0, P_{2}>0, R>0, Z_{j}$, $M_{j}>0(j=1,2,3,4), Q_{i}=\left(\begin{array}{cc}Q_{i 1} & Q_{i 2} \\ * & Q_{i 3}\end{array}\right)>0(i=1,2, \ldots, 6)$, positive diagonal matrices $T_{1}, T_{2}$, and matrices $N_{i}=\left(N_{i 1}^{T}, N_{i 2}^{T}\right.$, $\left.N_{i 3}^{T}\right)^{T}(i=1,2), S_{i} \geq 0(i=1,2,3,4)$ such that the following LMIs hold:

$$
\begin{aligned}
& P_{1}<\rho I, \\
& \Omega=\left(\Omega_{i, j}\right)_{22 \times 22}<0,
\end{aligned}
$$

where

$$
\begin{aligned}
\Omega_{1,1}= & A^{T} P_{1} A-P_{1}+\left(1+d_{2}\right) Q_{11}+Q_{21}+Q_{31} \\
& -\frac{1}{d_{M}} Z_{1}-\frac{d_{2}^{2}}{d_{3}} M_{1}-\frac{d_{M}^{2}}{d_{4}} M_{2} \\
& +N_{11}(A-I)+(A-I)^{T} N_{11}^{T}+\rho H_{1}, \\
\Omega_{1,2}= & -\frac{1}{d_{M}}\left(S_{1}^{T}-Z_{1}\right)+(A-I)^{T} N_{12}^{T}, \\
\Omega_{1,4}= & \frac{1}{d_{M}} S_{1}^{T}, \\
\Omega_{1,5}= & \left(1+d_{2}\right) Q_{12}+Q_{22}+Q_{32}-N_{11} \\
& +(A-I)^{T} N_{13}^{T}, \\
\Omega_{1,18}= & N_{11} B+A^{T} P_{1} B, \\
\Omega_{1,19}= & \frac{d_{2}}{d_{3}} M_{1}, \\
\Omega_{1,20}= & \frac{d_{M}}{d_{4}} M_{2},
\end{aligned}
$$

$$
\begin{aligned}
\Omega_{2,2}= & D^{T} P_{2} D+\rho H_{2}-Q_{11}-\frac{1}{d_{M}}\left(2 Z_{1}-S_{1}-S_{1}^{T}\right) \\
& -\frac{1}{d_{2}}\left(2 Z_{2}-S_{2}-S_{2}^{T}\right)
\end{aligned}
$$$$
\Omega_{2,3}=-\frac{1}{d_{2}}\left(S_{2}-Z_{2}\right) \text {, }
$$$$
\Omega_{2,4}=-\frac{1}{d_{M}}\left(-Z_{1}+S_{1}^{T}\right)-\frac{1}{d_{2}}\left(-Z_{2}+S_{2}\right),
$$$$
\Omega_{2,5}=-N_{12} \text {, }
$$$$
\Omega_{2,6}=-Q_{12} \text {, }
$$$$
\Omega_{2,9}=D^{T} P_{2} C+D^{T} N_{21}^{T} \text {, }
$$$$
\Omega_{2,10}=D^{T} N_{22}^{T} \text {, }
$$$$
\Omega_{2,13}=D^{T} N_{23}^{T} \text {, }
$$$$
\Omega_{2,18}=N_{12} B \text {, }
$$$$
\Omega_{3,3}=-Q_{21}-\frac{1}{d_{2}} Z_{2},
$$$$
\Omega_{3,4}=\frac{1}{d_{2}} S_{2}^{T},
$$$$
\Omega_{3,17}=-Q_{22} \text {, }
$$$$
\Omega_{4,4}=-Q_{31}-\frac{1}{d_{M}} Z_{1}-\frac{1}{d_{2}} Z_{2},
$$$$
\Omega_{4,8}=-Q_{32},
$$$$
\Omega_{5,5}=\left(1+d_{2}\right) Q_{13}+Q_{23}+Q_{33}+d_{M} Z_{1}+d_{2} Z_{2}
$$$$
+d_{3} M_{1}+d_{4} M_{2}-N_{13}-N_{13}^{T},
$$$$
\Omega_{5,18}=N_{13} B \text {, }
$$$$
\Omega_{6,6}=-Q_{13} \text {, }
$$$$
\Omega_{7,7}=-Q_{23} \text {, }
$$$$
\Omega_{8,8}=-Q_{33} \text {, }
$$$$
\Omega_{9,9}=C^{T} P_{2} C-P_{2}+\rho H_{3}+\left(1+\tau_{2}\right) Q_{41}+Q_{51}
$$$$
+Q_{61}-\frac{1}{\tau_{M}} Z_{3}-\frac{\tau_{2}^{2}}{\tau_{3}} M_{3}-\frac{\tau_{M}^{2}}{\tau_{4}} M_{4}
$$$$
+N_{21}(C-I)+(C-I)^{T} N_{21}^{T} \text {, }
$$$$
\Omega_{9,10}=-\frac{1}{\tau_{M}}\left(S_{3}^{T}-Z_{3}\right)+(C-I)^{T} N_{22}^{T},
$$$$
\Omega_{9,12}=\frac{1}{\tau_{M}} S_{3}^{T},
$$$$
\Omega_{9,13}=\left(1+\tau_{2}\right) Q_{42}+Q_{52}+Q_{62}-N_{21}
$$$$
+(C-I)^{T} N_{23}^{T} \text {, }
$$ 


$$
\begin{aligned}
& \Omega_{9,17}=L T_{1} \text {, } \\
& \Omega_{9,21}=\frac{\tau_{2}}{\tau_{3}} M_{3}, \\
& \Omega_{9,22}=\frac{\tau_{M}}{\tau_{4}} M_{4}, \\
& \Omega_{10,10}=-Q_{41}+\rho H_{4}-\frac{1}{\tau_{M}}\left(2 Z_{3}-S_{3}-S_{3}^{T}\right) \\
& -\frac{1}{\tau_{2}}\left(2 Z_{4}-S_{4}-S_{4}^{T}\right) \\
& \Omega_{10,11}=-\frac{1}{\tau_{2}}\left(S_{4}-Z_{4}\right), \\
& \Omega_{10,12}=-\frac{1}{\tau_{M}}\left(-Z_{3}+S_{3}^{T}\right)-\frac{1}{\tau_{2}}\left(-Z_{4}+S_{4}\right) \text {, } \\
& \Omega_{10,13}=-N_{22} \text {, } \\
& \Omega_{10,14}=-Q_{42} \text {, } \\
& \Omega_{10,18}=L T_{2}, \\
& \Omega_{11,11}=-Q_{51}-\frac{1}{\tau_{2}} Z_{4}, \\
& \Omega_{11,12}=\frac{1}{\tau_{2}} S_{4}^{T}, \\
& \Omega_{11,15}=-Q_{52} \text {, } \\
& \Omega_{12,12}=-Q_{61}-\frac{1}{\tau_{M}} Z_{3}-\frac{1}{\tau_{2}} Z_{4}, \\
& \Omega_{12,16}=-Q_{62} \text {, } \\
& \Omega_{13,13}=\left(1+\tau_{2}\right) Q_{43}+Q_{53}+Q_{63}+\tau_{M} Z_{3}+\tau_{2} Z_{4} \\
& +\tau_{3} M_{3}+\tau_{4} M_{4}-N_{23}-N_{23}^{T} \text {, } \\
& \Omega_{14,14}=-Q_{43} \text {, } \\
& \Omega_{15,15}=-Q_{53} \text {, } \\
& \Omega_{16,16}=-Q_{63} \text {, } \\
& \Omega_{17,17}=\left(1+\tau_{2}\right) R-T_{1}-T_{1}^{T}, \\
& \Omega_{18,18}=B^{T} P_{1} B-R-T_{2}-T_{2}^{T}, \\
& \Omega_{19,19}=-\frac{1}{d_{3}} M_{1}, \\
& \Omega_{20,20}=-\frac{1}{d_{4}} M_{2} \text {, } \\
& \Omega_{21,21}=-\frac{1}{\tau_{3}} M_{3} \\
& \Omega_{22,22}=-\frac{1}{\tau_{4}} M_{4}, \\
& d_{2}=d_{M}-d_{m} \text {, }
\end{aligned}
$$

$$
\begin{aligned}
& d_{3}=\frac{1}{2}\left(d_{M}-d_{m}\right)\left(d_{M}+d_{m}+1\right), \\
& d_{4}=\frac{1}{2} d_{M}\left(d_{M}+1\right), \\
& \tau_{2}=\tau_{M}-\tau_{m}, \\
& \tau_{3}=\frac{1}{2}\left(\tau_{M}-\tau_{m}\right)\left(\tau_{M}+\tau_{m}+1\right), \\
& \tau_{4}=\frac{1}{2} \tau_{M}\left(\tau_{M}+1\right)
\end{aligned}
$$

and the remaining terms are zero; then the considered system (7) is mean square asymptotically stable.

Proof. Set

$$
\begin{aligned}
& \lambda_{1}(k)=\left[\begin{array}{l}
x(k) \\
\eta_{1}(k)
\end{array}\right], \\
& \lambda_{2}(k)=\left[\begin{array}{c}
y(k) \\
\eta_{2}(k)
\end{array}\right], \\
& \eta_{1}(k)=x(k+1)-x(k), \\
& \eta_{2}(k)=y(k+1)-y(k) .
\end{aligned}
$$

Motivated by [26, 35], we construct the following LyapunovKrasovskii functional for the discrete-time stochastic GRN (7):

$$
V(k, x(k), y(k))=\sum_{i=1}^{6} V_{i}(k),
$$

where

$$
\begin{aligned}
V_{1}(k)= & x^{T}(k) P_{1} x(k)+y^{T}(k) P_{2} y(k), \\
V_{2}(k)= & \sum_{i=k-d(k)}^{k-1} \lambda_{1}^{T}(i) Q_{1} \lambda_{1}(i)+\sum_{i=k-d_{m}}^{k-1} \lambda_{1}^{T}(i) Q_{2} \lambda_{1}(i) \\
& +\sum_{i=k-d_{M}}^{k-1} \lambda_{1}^{T}(i) Q_{3} \lambda_{1}(i) \\
& +\sum_{j=-d_{M}+1}^{-d_{m}} \sum_{i=k+j}^{k-1} \lambda_{1}^{T}(i) Q_{1} \lambda_{1}(i),
\end{aligned}
$$




$$
\begin{aligned}
V_{3}(k)= & \sum_{i=k-\tau(k)}^{k-1} \lambda_{2}^{T}(i) Q_{4} \lambda_{2}(i)+\sum_{i=k-\tau_{m}}^{k-1} \lambda_{2}^{T}(i) Q_{5} \lambda_{2}(i) \\
& +\sum_{i=k-\tau_{M}}^{k-1} \lambda_{2}^{T}(i) Q_{6} \lambda_{2}(i) \\
& +\sum_{j=-\tau_{M}+1}^{-\tau_{m}} \sum_{i=k+j}^{k-1} \lambda_{2}^{T}(i) Q_{4} \lambda_{2}(i)
\end{aligned}
$$$$
V_{4}(k)=\sum_{i=k-\tau(k)}^{k-1} g^{T}(y(i)) R g(y(i))
$$$$
+\sum_{j=-\tau_{M}+1}^{-\tau_{m}} \sum_{i=k+j}^{k-1} g^{T}(y(i)) R g(y(i))
$$$$
V_{5}(k)=\sum_{j=-d_{M}}^{-1} \sum_{i=k+j}^{k-1} \eta_{1}^{T}(i) Z_{1} \eta_{1}(i)
$$$$
+\sum_{j=-d_{M}}^{-d_{m}-1} \sum_{i=k+j}^{k-1} \eta_{1}^{T}(i) Z_{2} \eta_{1}(i)
$$$$
+\sum_{j=-\tau_{M}}^{-1} \sum_{i=k+j}^{k-1} \eta_{2}^{T}(i) Z_{3} \eta_{2}(i)
$$$$
+\sum_{j=-\tau_{M}}^{-\tau_{m}-1} \sum_{i=k+j}^{k-1} \eta_{2}^{T}(i) Z_{4} \eta_{2}(i),
$$$$
V_{6}(k)=\sum_{l=-d_{M}}^{-d_{m}-1} \sum_{j=l}^{-1} \sum_{i=k+j}^{k-1} \eta_{1}^{T}(i) M_{1} \eta_{1}(i)
$$$$
+\sum_{l=-d_{m}}^{-1} \sum_{j=l}^{-1} \sum_{i=k+j}^{k-1} \eta_{1}^{T}(i) M_{2} \eta_{1}(i)
$$$$
+\sum_{l=-\tau_{M}}^{-\tau_{m}-1} \sum_{j=l}^{-1} \sum_{i=k+j}^{k-1} \eta_{2}^{T}(i) M_{3} \eta_{2}(i)
$$$$
+\sum_{l=-\tau_{m}}^{-1} \sum_{j=l}^{-1} \sum_{i=k+j}^{k-1} \eta_{2}^{T}(i) M_{4} \eta_{2}(i)
$$

Calculate the difference of $V(k)$ by defining $\Delta V(k)=V(k+$ $1)-V(k)$ along the solutions of (7) and taking mathematical expectation. It follows from Lemma 3 that

$$
\begin{aligned}
\mathbb{E} & {\left[\Delta V_{1}(k)\right]=\mathbb{E}\left[V_{1}(k+1)-V_{1}(k)\right] } \\
& =\mathbb{E}\left[x^{T}(k+1) P_{1} x(k+1)-x^{T}(k) P_{1} x(k)\right.
\end{aligned}
$$

$$
\begin{aligned}
& \left.+y^{T}(k+1) P_{2} y(k+1)-y^{T}(k) P_{2} y(k)\right] \\
& \leq \mathbb{E}\left[x^{T}(k)\left(A^{T} P_{1} A-P_{1}+\rho H_{1}\right) x(k)\right. \\
& +2 x^{T}(k) A^{T} P_{1} B g(y(k-\tau(k))) \\
& +x^{T}(k-d(k))\left(D^{T} P_{2} D+\rho H_{2}\right) x(k-d(k)) \\
& +y^{T}(k)\left(C^{T} P_{2} C-P_{2}+\rho H_{3}\right) y(k) \\
& +2 y^{T}(k) C^{T} P_{2} D x(k-d(k)) \\
& +y^{T}(k-\tau(k)) \rho H_{4} y(k-\tau(k)) \\
& \left.+g^{T}(y(k-\tau(k))) B^{T} P_{1} B g(y(k-\tau(k)))\right]
\end{aligned}
$$$$
\mathbb{E}\left[\Delta V_{2}(k)\right]=\mathbb{E}\left[V_{2}(k+1)-V_{2}(k)\right]
$$$$
\leq \mathbb{E}\left[\lambda_{1}^{T}(k)\left(Q_{1}+Q_{2}+Q_{3}\right) \lambda_{1}(k)\right.
$$$$
-\lambda_{1}^{T}(k-d(k)) Q_{1} \lambda_{1}(k-d(k))
$$$$
-\lambda_{1}^{T}\left(k-d_{m}\right) Q_{2} \lambda_{1}\left(k-d_{m}\right)
$$$$
-\lambda_{1}^{T}\left(k-d_{M}\right) Q_{3} \lambda_{1}\left(k-d_{M}\right)
$$$$
\left.+\sum_{i=k+1-d_{M}}^{k-d_{m}} \lambda_{1}^{T}(i) Q_{1} \lambda_{1}(i)\right]
$$$$
+\mathbb{E}\left[\sum _ { j = - d _ { M } + 1 } ^ { - d _ { m } } \left(\sum_{i=k+1+j}^{k} \lambda_{1}^{T}(i) Q_{1} \lambda_{1}(i)\right.\right.
$$$$
\left.\left.-\sum_{i=k+j}^{k-1} \lambda_{1}^{T}(i) Q_{1} \lambda_{1}(i)\right)\right]
$$$$
\leq \mathbb{E}\left[x^{T}(k)\left(Q_{11}+Q_{21}+Q_{31}\right) x(k)\right.
$$$$
+2 x^{T}(k)\left(Q_{12}+Q_{22}+Q_{32}\right) \eta_{1}(k)
$$$$
+\eta_{1}^{T}(k)\left(Q_{13}+Q_{23}+Q_{33}\right) \eta_{1}(k)
$$$$
-x^{T}(k-d(k)) Q_{11} x(k-d(k))
$$$$
-2 x^{T}(k-d(k)) Q_{12} \eta_{1}(k-d(k))
$$$$
-\eta_{1}^{T}(k-d(k)) Q_{13} \eta_{1}(k-d(k))
$$$$
-x^{T}\left(k-d_{m}\right) Q_{21} x\left(k-d_{m}\right)
$$$$
-2 x^{T}\left(k-d_{m}\right) Q_{22} \eta_{1}\left(k-d_{m}\right)
$$$$
-\eta_{1}^{T}\left(k-d_{m}\right) Q_{23} \eta_{1}\left(k-d_{m}\right)
$$$$
-x^{T}\left(k-d_{M}\right) Q_{31} x\left(k-d_{M}\right)
$$ 


$$
\begin{aligned}
& -2 x^{T}\left(k-d_{M}\right) Q_{32} \eta_{1}\left(k-d_{M}\right) \\
& \mathbb{E}\left[\Delta V_{5}(k)\right]=\mathbb{E}\left[V_{5}(k+1)-V_{5}(k)\right] \\
& -\eta_{1}^{T}\left(k-d_{M}\right) Q_{33} \eta_{1}\left(k-d_{M}\right) \\
& +\sum_{i=k+1-d_{M}}^{k-d_{m}} \lambda_{1}^{T}(i) Q_{1} \lambda_{1}(i) \\
& +d_{2}\left(x^{T}(k) Q_{11} x(k)+2 x^{T}(k) Q_{12} \eta_{1}(k)\right. \\
& \left.+\eta^{T}(k) Q_{13} \eta_{1}(k)\right) \\
& \left.-\sum_{i=k+1-d_{M}}^{k-d_{m}} \lambda_{1}^{T}(i) Q_{1} \lambda_{1}(i)\right], \\
& \mathbb{E}\left[\Delta V_{3}(k)\right]=\mathbb{E}\left[V_{3}(k+1)-V_{3}(k)\right] \\
& \leq \mathbb{E}\left[y^{T}(k)\left(Q_{41}+Q_{51}+Q_{61}\right) y(k)\right. \\
& +2 y^{T}(k)\left(Q_{42}+Q_{52}+Q_{62}\right) \eta_{2}(k) \\
& +\eta_{2}^{T}(k)\left(Q_{43}+Q_{53}+Q_{63}\right) \eta_{2}(k) \\
& \text { - } y^{T}(k-\tau(k)) Q_{41} x(k-d(k)) \\
& -2 y^{T}(k-\tau(k)) Q_{42} \eta_{2}(k-\tau(k)) \\
& -\eta_{2}^{T}(k-\tau(k)) Q_{43} \eta_{2}(k-\tau(k)) \\
& -y^{T}\left(k-\tau_{m}\right) Q_{51} x\left(k-\tau_{m}\right) \\
& -2 y^{T}\left(k-\tau_{m}\right) Q_{52} \eta_{2}\left(k-\tau_{m}\right) \\
& -\eta_{2}^{T}\left(k-\tau_{m}\right) Q_{53} \eta_{2}\left(k-\tau_{m}\right) \\
& -y^{T}\left(k-\tau_{M}\right) Q_{61} x\left(k-\tau_{M}\right) \\
& -2 y^{T}\left(k-\tau_{M}\right) Q_{62} \eta_{2}\left(k-\tau_{M}\right) \\
& -\eta_{2}^{T}\left(k-\tau_{M}\right) Q_{63} \eta_{2}\left(k-\tau_{M}\right) \\
& +\sum_{i=k+1-\tau_{M}}^{k-\tau_{m}} \lambda_{2}^{T}(i) Q_{4} \lambda_{2}(i) \\
& +\tau_{2}\left(y^{T}(k) Q_{41} y(k)\right. \\
& \left.+2 y^{T}(k) Q_{42} \eta_{2}(k)+\eta_{2}^{T}(k) Q_{43} \eta_{2}(k)\right) \\
& \left.-\sum_{i=k+1-\tau_{M}}^{k-\tau_{m}} \lambda_{2}^{T}(i) Q_{4} \lambda_{2}(i)\right], \\
& \mathbb{E}\left[\Delta V_{4}(k)\right]=\mathbb{E}\left[V_{4}(k+1)-V_{4}(k)\right] \\
& \leq \mathbb{E}\left[\left(1+\tau_{2}\right) g^{T}(y(k)) \operatorname{Rg}(y(k))\right. \\
& \left.-g^{T}(y(k-\tau(k))) \operatorname{Rg}(y(k-\tau(k)))\right] \text {, } \\
& =\mathbb{E}\left[\sum _ { j = - d _ { M } } ^ { - 1 } \left(\sum_{i=k+1+j}^{k} \eta_{1}^{T}(i) Z_{1} \eta_{1}(i)\right.\right. \\
& -\sum_{i=k+j}^{k-1} \eta_{1}^{T}(i) Z_{1} \eta_{1}(i) \\
& +\sum_{j=-d_{M}}^{-d_{m}-1}\left(\sum_{i=k+1+j}^{k} \eta_{1}^{T}(i) Z_{2} \eta_{1}(i)\right. \\
& -\sum_{i=k+j}^{k-1} \eta_{1}^{T}(i) Z_{2} \eta_{1}(i) \\
& +\sum_{j=-\tau_{M}}^{-1}\left(\sum_{i=k+1+j}^{k} \eta_{2}^{T}(i) Z_{3} \eta_{2}(i)\right. \\
& -\sum_{i=k+j}^{k-1} \eta_{2}^{T}(i) Z_{3} \eta_{2}(i) \\
& +\sum_{j=-\tau_{M}}^{-\tau_{m}-1}\left(\sum_{i=k+1+j}^{k} \eta_{2}^{T}(i) Z_{4} \eta_{2}(i)\right. \\
& \left.\left.-\sum_{i=k+j}^{k-1} \eta_{2}^{T}(i) Z_{4} \eta_{2}(i)\right)\right] \\
& =\mathbb{E}\left[d_{M} \eta_{1}^{T}(k) Z_{1} \eta_{1}(k)\right. \\
& -\sum_{i=k-d_{M}}^{k-1} \eta_{1}^{T}(i) Z_{1} \eta_{1}(i) \\
& +d_{2} \eta_{1}^{T}(k) Z_{2} \eta_{1}(k) \\
& -\sum_{i=k-d_{M}}^{k-d_{m}-1} \eta_{1}^{T}(i) Z_{2} \eta_{1}(i) \\
& +\tau_{M} \eta_{2}^{T}(k) Z_{3} \eta_{2}(k) \\
& -\sum_{i=k-\tau_{M}}^{k-1} \eta_{2}^{T}(i) Z_{3} \eta_{2}(i) \\
& +\tau_{2} \eta_{2}^{T}(k) Z_{4} \eta_{2}(k) \\
& \left.-\sum_{i=k-\tau_{M}}^{k-\tau_{m}-1} \eta_{2}^{T}(i) Z_{4} \eta_{2}(i)\right]
\end{aligned}
$$




$$
\begin{aligned}
& \mathbb{E}\left[\Delta V_{6}(k)\right]=\mathbb{E}\left[V_{6}(k+1)-V_{6}(k)\right] \\
& =\mathbb{E}\left[\sum _ { l = - d _ { M } } ^ { - d _ { m } - 1 } \sum _ { j = l } ^ { - 1 } \left(\sum_{i=k+1+j}^{k} \eta_{1}^{T}(i) M_{1} \eta_{1}(i)\right.\right. \\
& \left.-\sum_{i=k+j}^{k-1} \eta_{1}^{T}(i) M_{1} \eta_{1}(i)\right) \\
& +\sum_{l=-d_{M}}^{-1} \sum_{j=l}^{-1}\left(\sum_{i=k+1+j}^{k} \eta_{1}^{T}(i) M_{2} \eta_{1}(i)\right. \\
& \left.-\sum_{i=k+j}^{k-1} \eta_{1}^{T}(i) M_{2} \eta_{1}(i)\right) \\
& +\sum_{l=-\tau_{M}}^{-\tau_{m}-1} \sum_{j=l}^{-1}\left(\sum_{i=k+1+j}^{k} \eta_{2}^{T}(i) M_{3} \eta_{2}(i)\right. \\
& \left.-\sum_{i=k+j}^{k-1} \eta_{2}^{T}(i) M_{3} \eta_{2}(i)\right) \\
& +\sum_{l=-\tau_{M}}^{-1} \sum_{j=l}^{-1}\left(\sum_{i=k+1+j}^{k} \eta_{2}^{T}(i) M_{4} \eta_{2}(i)\right. \\
& \left.\left.-\sum_{i=k+j}^{k-1} \eta_{2}^{T}(i) M_{4} \eta_{2}(i)\right)\right] \\
& =\mathbb{E}\left[d_{3} \eta_{1}^{T}(k) M_{1} \eta_{1}(k)\right. \\
& -\sum_{l=-d_{M}}^{-d_{m}-1} \sum_{j=k+l}^{k-1} \eta_{1}^{T}(j) M_{1} \eta_{1}(j)+d_{4} \eta_{1}^{T}(k) M_{2} \eta_{1}(k) \\
& -\sum_{i=k-d_{M}}^{k-1} \eta_{1}^{T}(i) Z_{1} \eta_{1}(i)=-\sum_{i=k-d_{M}}^{k-d(k)-1} \eta_{1}^{T}(i) Z_{1} \eta_{1}(i) \\
& -\sum_{i=k-d(k)}^{k-1} \eta_{1}^{T}(i) Z_{1} \eta_{1}(i) \\
& \leq-\frac{1}{d_{M}}\left[\frac{d_{M}}{d_{M}-d(k)}\left(\sum_{i=k-d_{M}}^{k-d(k)-1} \eta_{1}(i)\right)^{T}\right. \\
& \cdot Z_{1}\left(\sum_{i=k-d_{M}}^{k-d(k)-1} \eta_{1}(i)\right)+\frac{d_{M}}{d(k)}\left(\sum_{i=k-d(k)}^{k-1} \eta_{1}(i)\right)^{T} \\
& \left.\cdot Z_{1}\left(\sum_{i=k-d(k)}^{k-1} \eta_{1}(i)\right)\right] \\
& =-\frac{1}{d_{M}}\left[\frac{d_{M}}{d_{M}-d(k)}(x(k-d(k))\right. \\
& \left.-x\left(k-d_{M}\right)\right)^{T} Z_{1}\left(x(k-d(k))-x\left(k-d_{M}\right)\right) \\
& +\frac{d_{M}}{d(k)}(x(k)-x(k-d(k)))^{T} Z_{1}(x(k) \\
& -x(k-d(k)))] \\
& \leq-\frac{1}{d_{M}}\left[\left(\begin{array}{c}
x(k-d(k))-x\left(k-d_{M}\right) \\
x(k)-x(k-d(k))
\end{array}\right)^{T}\right. \\
& \left.\cdot\left(\begin{array}{cc}
Z_{1} & S_{1} \\
S_{1}^{T} & Z_{1}
\end{array}\right)\left(\begin{array}{c}
x(k-d(k))-x\left(k-d_{M}\right) \\
x(k)-x(k-d(k))
\end{array}\right)\right] .
\end{aligned}
$$$$
\left.-\sum_{l=-d_{M}}^{-1} \sum_{j=k+l}^{k-1} \eta_{1}^{T}(j) M_{2} \eta_{1}(j)\right]
$$$$
+\mathbb{E}\left[\tau_{3} \eta_{2}^{T}(k) M_{3} \eta_{2}(k)\right.
$$$$
-\sum_{l=-\tau_{M}}^{-\tau_{m}-1} \sum_{j=k+l}^{k-1} \eta_{2}^{T}(j) M_{3} \eta_{2}(j)+\tau_{4} \eta_{2}^{T}(k) M_{4} \eta_{2}(k)
$$$$
\left.-\sum_{l=-\tau_{M}}^{-1} \sum_{j=k+l}^{k-1} \eta_{2}^{T}(j) M_{4} \eta_{2}(j)\right] .
$$

Similarly, we have

$$
\begin{aligned}
& -\sum_{i=k-d_{M}}^{k-d_{m}-1} \eta_{1}^{T}(i) Z_{2} \eta_{1}(i) \\
& \leq-\frac{1}{d_{2}}\left[\left(\begin{array}{c}
x(k-d(k))-x\left(k-d_{M}\right) \\
x\left(k-d_{m}\right)-x(k-d(k))
\end{array}\right)^{T}\right. \\
& \left.\cdot\left(\begin{array}{cc}
Z_{2} & S_{2} \\
S_{2}^{T} & Z_{2}
\end{array}\right)\left(\begin{array}{c}
x(k-d(k))-x\left(k-d_{M}\right) \\
x\left(k-d_{m}\right)-x(k-d(k))
\end{array}\right)\right],
\end{aligned}
$$




$$
\begin{aligned}
& -\sum_{i=k-\tau_{M}}^{k-1} \eta_{2}^{T}(i) Z_{3} \eta_{2}(i) \\
& \leq-\frac{1}{\tau_{M}}\left[\left(\begin{array}{c}
y(k-\tau(k))-y\left(k-\tau_{M}\right) \\
y(k)-y(k-\tau(k))
\end{array}\right)^{T}\right. \\
& \left.\cdot\left(\begin{array}{cc}
Z_{3} & S_{3} \\
S_{3}^{T} & Z_{3}
\end{array}\right)\left(\begin{array}{c}
y(k-\tau(k))-y\left(k-\tau_{M}\right) \\
y(k)-y(k-\tau(k))
\end{array}\right)\right], \\
& -\sum_{i=k-\tau_{M}}^{k-\tau_{m}-1} \eta_{2}^{T}(i) Z_{4} \eta_{2}(i) \\
& \leq-\frac{1}{\tau_{2}}\left[\left(\begin{array}{c}
y(k-\tau(k))-y\left(k-\tau_{M}\right) \\
y\left(k-\tau_{m}\right)-y(k-\tau(k))
\end{array}\right)^{T}\right. \\
& \left.\cdot\left(\begin{array}{ll}
Z_{4} & S_{4} \\
S_{4}^{T} & Z_{4}
\end{array}\right)\left(\begin{array}{c}
y(k-\tau(k))-y\left(k-\tau_{M}\right) \\
y\left(k-\tau_{m}\right)-y(k-\tau(k))
\end{array}\right)\right], \\
& -\sum_{l=-\tau_{M}}^{-\tau_{m}-1} \sum_{j=k+l}^{k-1} \eta_{2}^{T}(j) M_{3} \eta_{2}(j) \\
& \leq-\frac{1}{\tau_{3}}\left[\tau_{2} y(k)-\sum_{i=k-\tau_{M}}^{k-\tau_{m}-1} y(i)\right]^{T} \\
& \cdot M_{3}\left[\tau_{2} y(k)-\sum_{i=k-\tau_{M}}^{k-\tau_{m}-1} y(i)\right] \text {, } \\
& -\sum_{l=-\tau_{M}}^{-1} \sum_{j=k+l}^{k-1} \eta_{2}^{T}(j) M_{4} \eta_{2}(j) \\
& \leq-\frac{1}{\tau_{4}}\left[\tau_{M} y(k)-\sum_{i=k-\tau_{M}}^{k-1} y(i)\right]^{T} \\
& \cdot M_{4}\left[\tau_{M} y(k)-\sum_{i=k-\tau_{M}}^{k-1} y(i)\right] \text {, }
\end{aligned}
$$

where $d_{2}$ and $\tau_{2}$ are defined in Theorem 5 .

By applying Lemma 3 , for $M_{j}>0(j=1,2,3,4)$, the following inequalities can be established:

$$
\begin{aligned}
& -\sum_{l=-d_{M}}^{-d_{m}-1} \sum_{j=k+l}^{k-1} \eta_{1}^{T}(j) M_{1} \eta_{1}(j) \\
& \leq-\frac{1}{d_{3}}\left[\sum_{l=-d_{M}}^{-d_{m}-1} \sum_{j=k+l}^{k-1} \eta_{1}^{T}(j) M_{1} \sum_{l=-d_{M}}^{-d_{m}-1} \sum_{j=k+l}^{k-1} \eta_{1}(j)\right] \\
& \leq-\frac{1}{d_{3}}\left[d_{2} x(k)-\sum_{i=k-d_{M}}^{k-d_{m}-1} x(i)\right]^{T} \\
& \cdot M_{1}\left[d_{2} x(k)-\sum_{i=k-d_{M}}^{k-d_{m}-1} x(i)\right] \\
& -\sum_{l=-d_{M}}^{-1} \sum_{j=k+l}^{k-1} \eta_{1}^{T}(j) M_{2} \eta_{1}(j) \\
& \leq-\frac{1}{d_{4}}\left[d_{M} x(k)-\sum_{i=k-d_{M}}^{k-1} x(i)\right]^{T} \\
& \cdot M_{2}\left[d_{M} x(k)-\sum_{i=k-d_{M}}^{k-1} x(i)\right]
\end{aligned}
$$

where $d_{3}, d_{4}, \tau_{3}$, and $\tau_{4}$ are defined in Theorem 5. From Assumption 1, for diagonal matrices $T_{1}, T_{2}>0$ and a known matrix $L=\operatorname{diag}\left\{l_{1}, l_{2}, \ldots, l_{n}\right\}>0$, it is not difficult to see that the following inequalities

$$
\begin{aligned}
& 2 g^{T}(y(k)) T_{1} g(y(k))-2 y^{T}(k) L T_{1} g(y(k)) \leq 0, \\
& 2 g^{T}(y(k-\tau(k))) T_{2} g(y(k-\tau(k))) \\
& \quad-2 y^{T}(k-\tau(k)) L T_{2} g(y(k-\tau(k))) \leq 0
\end{aligned}
$$

hold.

In addition, we get

$$
\begin{aligned}
\mathbb{E}\left[\eta_{1}(k)\right] & =\mathbb{E}[x(k+1)-x(k)] \\
& =\mathbb{E}[(A-I) x(k)+B g(y(k-\tau(k)))], \\
\mathbb{E}\left[\eta_{2}(k)\right] & =\mathbb{E}[y(k+1)-y(k)] \\
& =\mathbb{E}[(C-I) y(k)+D x(k-d(k))] .
\end{aligned}
$$

Then, for any matrices $N_{1}=\left[N_{11}^{T}, N_{12}^{T}, N_{13}^{T}\right]^{T}$ and $N_{2}=$ $\left[N_{21}^{T}, N_{22}^{T}, N_{23}^{T}\right]^{T}$, we have

$\mathbb{E}\left[2 \alpha_{1}(k)\right.$

$$
\begin{aligned}
& \left.\cdot N_{1}\left((A-I) x(k)+B g(y(k-\tau(k)))-\eta_{1}(k)\right)\right] \\
& =0
\end{aligned}
$$

$$
\begin{aligned}
& \mathbb{E}\left[2 \alpha_{2}(k)\right. \\
& \left.\cdot N_{2}\left((C-I) y(k)+D x(k-d(k))-\eta_{2}(k)\right)\right]=0,
\end{aligned}
$$

where

$$
\begin{aligned}
& \alpha_{1}^{T}(k)=\left(x^{T}(k), x^{T}(k-d(k)), \eta_{1}^{T}(k)\right), \\
& \alpha_{2}^{T}(k)=\left(y^{T}(k), y^{T}(k-\tau(k)), \eta_{2}^{T}(k)\right) .
\end{aligned}
$$


Now, combining (19), (20), (21), (22), (23), and (25), we obtain the following inequality:

$$
\mathbb{E}[\Delta V(k)] \leq \mathbb{E}\left[\alpha^{T}(k) \Omega \alpha(k)\right]
$$

with

$$
\begin{aligned}
& \alpha^{T}(k)=\left(x^{T}(k), x^{T}(k-d(k)), x^{T}\left(k-d_{m}\right),\right. \\
& x^{T}\left(k-d_{M}\right), \eta_{1}^{T}(k), \eta_{1}^{T}(k-d(k)), \eta_{1}^{T}\left(k-d_{m}\right), \\
& \eta_{1}^{T}\left(k-d_{M}\right), y^{T}(k), y^{T}(k-\tau(k)), y^{T}\left(k-\tau_{m}\right), \\
& y^{T}\left(k-\tau_{M}\right), \eta_{2}^{T}(k), \eta_{2}^{T}(k-\tau(k)), \eta_{2}^{T}\left(k-\tau_{m}\right), \\
& \eta_{2}^{T}\left(k-\tau_{M}\right), g^{T}(y(k)), g^{T}(y(k-\tau(k))), \\
& \left.\quad \sum_{i=k-d_{m}-1}^{k} x^{T}(i), \sum_{i=k-d_{M}}^{k-1} x^{T}(i), \sum_{i=k-\tau_{M}}^{k-\tau_{m}-1} y^{T}(i), \sum_{i=k-\tau_{M}}^{k-1} y^{T}(i)\right) .
\end{aligned}
$$

According to LMIs (14), one has $\Omega<0$. Hence, we have $\mathbb{E}[\Delta V(k)] \leq 0$. Thus, the considered stochastic GRN is asymptotically stable in mean square sense, which ends the proof of this theorem.

Remark 6. Note that a novel Lyapunov-Krasovskii functional has been proposed in [26] including some triple summation terms, where some new yet effective robust stabilization and $H_{\infty}$ control schemes have been given for discrete uncertain GRNs with time-varying delays and disturbances. In order to further reduce the conservatism induced by the time delays, it is worth mentioning that a new Lyapunov-Krasovskii functional has been constructed as in Theorem 5 motivated by the efficient results in $[26,35]$. Moreover, the discretetime Jensen inequality lemma has been used in Theorem 5 to deal with the summation terms (20) and (21) as in [26], where more information of the time-varying delays has been utilized in our main results. In addition, as pointed out in [34], by using the lower bounds theorem and the discretetime Jensen inequality, less conservative stability criteria can be obtained compared to the one where the discretetime Jensen inequality is used only. Hence, based on the combination of the discrete-time Jensen inequality, the lower bounds theorem, and the free-weighting matrix approach, some useful information of the time delays is considered and then a new stability condition is proposed for the addressed delayed GRNs and the conservatism from the time-varying delays is further reduced.

Remark 7. The standard LMI system has a polynomial-time complexity, which is bounded by $O(M N)$, where $M$ is the total row size of the LMI system and $N$ is the total number of scalar decision variables. For example, let us consider the proposed stability criterion for the addressed GRNs (as described in Theorem 5); we have $M=22 n$ and $N=1+$ $11 n(n+1) / 2+6\left(n(n+1)+n^{2}\right)+2 n+6 n^{2}+4 n^{2}=(1 / 2)\left(55 n^{2}+\right.$ $27 n+2)$. Therefore, the computational complexity of the LMI-based stability criterion can be represented as $O\left(605 n^{3}+\right.$ $\left.297 n^{2}+22 n\right)$.

\section{Numerical Simulations}

In this section, we provide two numerical examples to illustrate the effectiveness and correctness of the main results.

Example 1. Consider the five-node GRN (7) with its parameters borrowed from [21]:

$$
\begin{aligned}
& A=C=\operatorname{diag}\{0.8,0.7,0.6,0.4,0.2\}, \\
& D=0.1 I, \\
& H_{1}=H_{2}=H_{3}=H_{4}=I, \\
& B=0.5 \times\left(\begin{array}{ccccc}
0 & -1 & 1 & 0 & 0 \\
-1 & 0 & 0 & 1 & 1 \\
0 & 1 & 0 & 0 & 0 \\
1 & -1 & 0 & 0 & 0 \\
0 & 0 & 0 & 1 & 0
\end{array}\right) .
\end{aligned}
$$

Letting the nonlinear regulatory function be $g(y)=$ $y^{2} /\left(1+y^{2}\right)$, then it is easy to see

$$
L=\operatorname{diag}\{0.65,0.65,0.65,0.65,0.65\} \text {. }
$$

Moreover, assume $d_{m}=4, d_{M}=6, \tau_{m}=1, \tau_{M}=3$. By using the LMIs control toolbox in Matlab to solve the LMIs in Theorem 5, we can see that they are feasible with parts of the obtained parameters as follows:

$P_{1}=\left(\begin{array}{ccccc}18.7632 & -2.1967 & 9.9367 & -3.0025 & 0.6567 \\ -2.1967 & 16.4957 & 2.6272 & 8.6953 & -5.5338 \\ 9.9367 & 2.6272 & 38.3963 & 10.0564 & -0.9914 \\ -3.0025 & 8.6953 & 10.0564 & 25.6204 & -5.6826 \\ 0.6567 & -5.5338 & -0.9914 & -5.6826 & 48.6707\end{array}\right)$,

$P_{2}$

$$
\begin{gathered}
=\left(\begin{array}{ccccc}
47.7466 & -5.0327 & 4.1261 & -9.5940 & -7.6801 \\
-5.0327 & 64.7246 & -15.8688 & 2.8260 & -1.5562 \\
4.1261 & -15.8688 & 74.5614 & -0.0815 & -2.7697 \\
-9.5940 & 2.8260 & -0.0815 & 78.7523 & 11.4312 \\
-7.6801 & -1.5562 & -2.7697 & 11.4312 & 79.3701
\end{array}\right), \\
R=\left(\begin{array}{ccccc}
0.7715 & -0.0504 & 0.1792 & -0.1834 & -0.1930 \\
-0.0504 & 1.6419 & -0.1470 & 0.1093 & -0.1013 \\
0.1792 & -0.1470 & 2.4800 & 0.0101 & -0.0965 \\
-0.1834 & 0.1093 & 0.0101 & 2.7531 & 0.2216 \\
-0.1930 & -0.1013 & -0.0965 & 0.2216 & 2.5567
\end{array}\right) .
\end{gathered}
$$

Therefore, according to Theorem 5, GRN (7) with timevarying delays and stochastic disturbances is mean square asymptotically stable. Based on the parameter mentioned above, the trajectories of the mRNA and protein concentrations are plotted in Figures 1 and 2, respectively. 


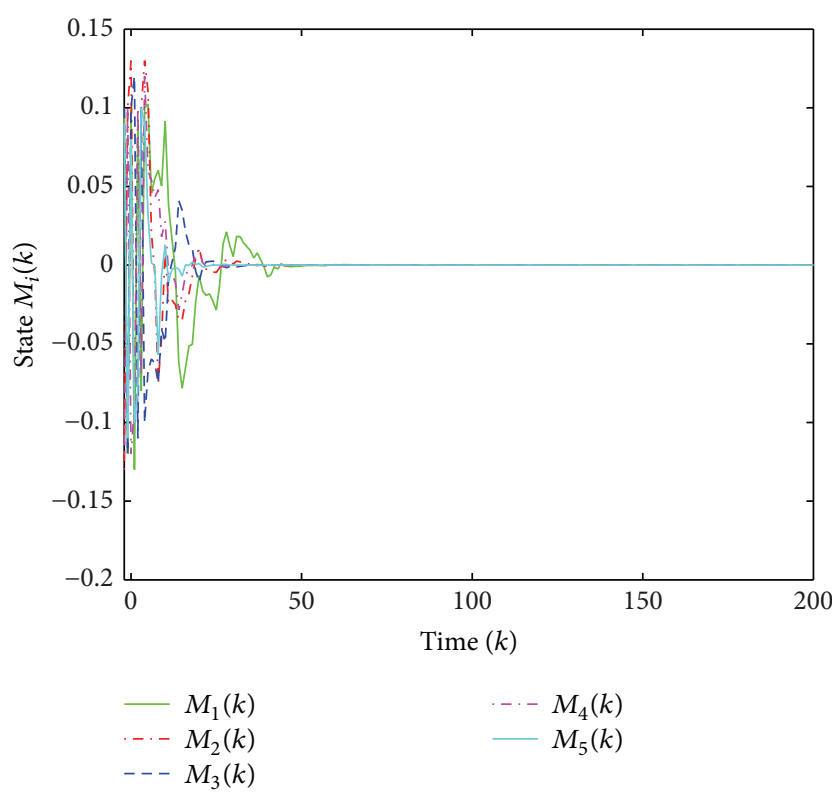

FIgURE 1: The trajectories of the mRNA concentrations.

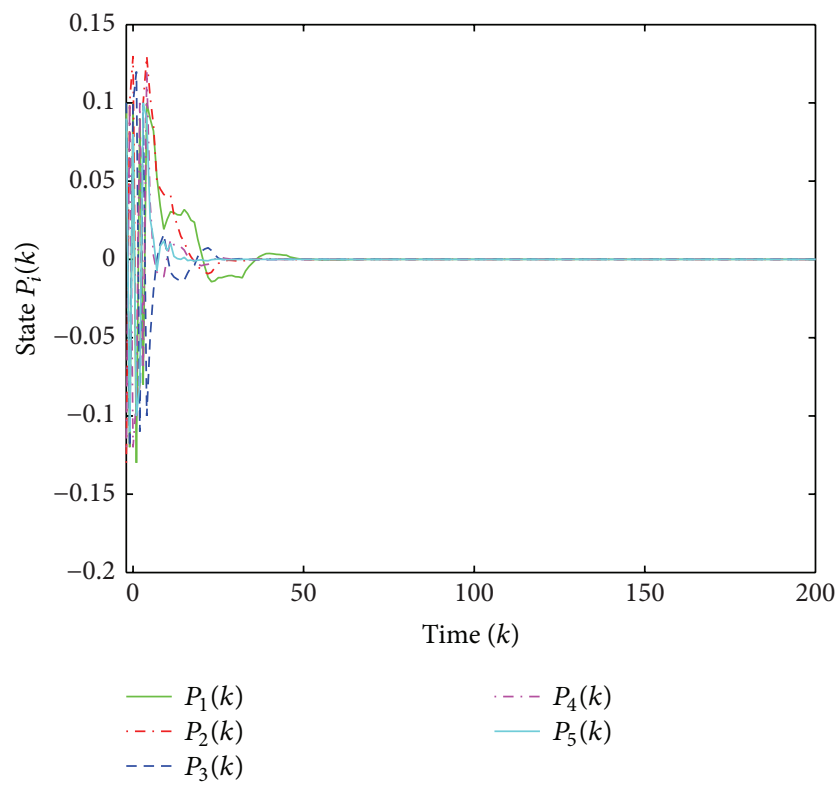

FIGURE 2: The trajectories of the protein concentrations.

Example 2. Consider the five-node GRN (7) with the other case:

$$
\begin{aligned}
A & =C=\operatorname{diag}\{0.8,0.7,0.6,0.4,0.2\}, \\
H_{1} & =H_{2}=0, \\
H_{3} & =H_{4}=I ;
\end{aligned}
$$

the delay $d(k)$ is unknown, and the other parameters are listed as in Example 1. According to the different lower bounds of time delays, the delays upper bounds for guaranteeing the asymptotical stability of the addressed GRN are obtained as in Table 1 by using Theorem 5, Theorem 3 in [20], and
TABLE 1: Admissible upper bound $d_{M}$ with different $d_{m}$.

\begin{tabular}{lcccccc}
\hline$d_{m}$ & 2 & 4 & 6 & 8 & 10 & 12 \\
\hline Theorem 3 in [20] & 4 & 6 & 8 & 9 & 11 & 14 \\
Theorem 1 in [21] & 9 & 11 & 13 & 15 & 17 & 19 \\
Theorem 5 & 14 & 15 & 17 & 19 & 21 & 23 \\
\hline
\end{tabular}

Theorem 1 in [21], respectively. It should be pointed out that Theorem 1 in [20] is infeasible when setting $H_{3}=H_{4}=0$, the feedback regulation delay $\tau(k)$, and the translation delay $d(k)$ satisfying $2 \leq \tau(k) \leq 20$ and $1 \leq d(k) \leq 22$, respectively; however, it is easy to see that system (7) is asymptotically stable by using Theorem 5 proposed in this paper. Therefore, the newly proposed result has less conservatism than the results in $[20,21]$.

\section{Conclusion}

In the paper, we have investigated the stability analysis problem for discrete-time GRNs with interval time-varying delays and stochastic disturbances. By utilizing a new augmented Lyapunov-Krasovskii functional which contains some triple summation terms, the discrete-time Jensen inequality, and the lower bound lemma, an improved delay-dependent stability criterion has been given in terms of LMIs to guarantee the mean square asymptotic stability for the addressed discrete-time GRNs. The effectiveness and advantage of the proposed method have been illustrated by utilizing two numerical examples. Based on the proposed results, we can extend the idea of this paper to other system models with Markovian jumping parameters [35, 36], time-varying parameters [37, 38], stochastic uncertainties [39, 40], and stochastic nonlinearities [41-43]. Moreover, the problems of the robust state estimation and $H_{\infty}$ filtering for discrete GRNs with different scourers noises as in [44-46] will be discussed in our future research.

\section{Competing Interests}

The authors declare that there is no conflict of interests regarding the publication of this paper.

\section{Acknowledgments}

This work was supported in part by the National Natural Science Foundation of China under Grants 11401141 and 11271103, Heilongjiang Provincial Natural Science Foundation for Youths under Grant QC2013C001, Heilongjiang Provincial Postdoctoral Science Foundation under Grant LBH-Z15098, and Natural Science Foundation of Heilongjiang Educational Committee under Grant 12531099.

\section{References}

[1] I. Shmulevich, E. R. Dougherty, S. Kim, and W. Zhang, "Probabilistic Boolean networks: a rule-based uncertainty model for 
gene regulatory networks," Bioinformatics, vol. 18, no. 2, pp. 261274, 2002.

[2] X. Zhang, A. Yu, and G. Zhang, "M-matrix-based delay-rangedependent global asymptotical stability criterion for genetic regulatory networks with time-varying delays," Neurocomputing, vol. 113, no. 7, pp. 8-15, 2013.

[3] I. Ivanov and E. R. Dougherty, "Modelling genetic regulatory networks: continuous or discrete," Journal of Biological Systems, vol. 2, no. 2, pp. 219-229, 2011.

[4] G. Chesi and Y. S. Hung, "Stability analysis of uncertain genetic sum regulatory networks," Automatica, vol. 44, no. 9, pp. 22982305, 2008.

[5] J. Liang, J. Lam, and Z. Wang, "State estimation for Markov-type genetic regulatory networks with delays and uncertain mode transition rates," Physics Letters A, vol. 373, no. 47, pp. 43284337, 2009.

[6] Y. Yao, J. Liang, and J. Cao, "Robust stability of Markovian jumping genetic regulatory networks with disturbance attenuation," Asian Journal of Control, vol. 13, no. 5, pp. 655-666, 2011.

[7] Y. Liu and H. Wu, "Analysis on global asymptotical stability of genetic regulatory networks with time-varying delays via convex combination method," Mathematical Problems in Engineering, vol. 2015, Article ID 303918, 5 pages, 2015.

[8] A. Liu, L. Yu, W. Zhang, and B. Chen, " $H_{\infty}$ filtering for discrete-time genetic regulatory networks with random delays," Mathematical Biosciences, vol. 239, no. 1, pp. 97-105, 2012.

[9] L. Chen and K. Aihara, "Stability of genetic regulatory networks with time delay," IEEE Transactions on Circuits and Systems. I. Fundamental Theory and Applications, vol. 49, no. 5, pp. 602608, 2002.

[10] J. Hu, Z. Wang, B. Shen, and H. Gao, "Gain-constrained recursive filtering with stochastic nonlinearities and probabilistic sensor delays," IEEE Transactions on Signal Processing, vol. 61, no. 5, pp. 1230-1238, 2013.

[11] J. Cao and F. Ren, "Exponential stability of discrete-time genetic regulatory networks with delays," IEEE Transactions on Neural Networks, vol. 19, no. 3, pp. 520-523, 2008.

[12] J. Hu, Z. Wang, and H. Gao, Nonlinear Stochastic Systems with Network-Induced Phenomena: Recursive Filtering and SlidingMode Design, Springer, London, UK, 2015.

[13] Y. Yu, H. Dong, Z. Wang, W. Ren, and F. E. Alsaadi, "Design of non-fragile state estimators for discrete time-delayed neural networks with parameter uncertainties," Neurocomputing, vol. 182, pp. 18-24, 2016.

[14] J. Hu, D. Chen, and J. Du, "State estimation for a class of discrete nonlinear systems with randomly occurring uncertainties and distributed sensor delays," International Journal of General Systems, vol. 43, no. 3-4, pp. 387-401, 2014.

[15] K. Mathiyalagan, J. H. Park, and R. Sakthivel, "Novel results on robust finite-time passivity for discrete-time delayed neural networks," Neurocomputing, vol. 177, pp. 585-593, 2016.

[16] J. Hu, Z. Wang, H. Dong, and H. Gao, "Recent advances on recursive filtering and sliding mode design for networked nonlinear stochastic systems: a survey," Mathematical Problems in Engineering, vol. 2013, Article ID 646059, 12 pages, 2013.

[17] F. Ren and J. Cao, "Asymptotic and robust stability of genetic regulatory networks with time-varying delays," Neurocomputing, vol. 71, no. 4-6, pp. 834-842, 2008.

[18] H. Wu, X. Liao, W. Feng, S. Guo, and W. Zhang, "Robust stability for uncertain genetic regulatory networks with interval timevarying delays," Information Sciences, vol. 180, no. 18, pp. 35323545, 2010.
[19] C. Y. Lu, Y. C. Hsiao, C. H. Liu, C. H. Hsu, K. Y. Chang, and S. J. Cheng, "Robust stability for discrete-time uncertain genetic regulatory networks with infinite-distributed delay," in Proceedings of the 2nd International Symposium on Computer, Consumer and Control (IS3C '14), pp. 395-398, IEEE, Taichung, Taiwan, June 2014.

[20] Q. Ma, S. Xu, Y. Zou, and J. Lu, "Robust stability for discretetime stochastic genetic regulatory networks," Nonlinear Analysis: Real World Applications, vol. 12, no. 5, pp. 2586-2595, 2011.

[21] Y. He, J. Zeng, M. Wu, C.-K. Zhang, and Y. Zhang, "Stability for discrete-time genetic regulatory networks with time-varying interval delays," Control Theory and Applications, vol. 29, no. 11, pp. 1465-1470, 2012.

[22] D. Zhang, H. Song, L. Yu, Q.-G. Wang, and C. Ong, "Set-values filtering for discrete time-delay genetic regulatory networks with time-varying parameters," Nonlinear Dynamics, vol. 69, no. 1-2, pp. 693-703, 2012.

[23] R. Anbuvithya, K. Mathiyalagan, R. Sakthivel, and P. Prakash, "Sampled-data state estimation for genetic regulatory networks with time-varying delays," Neurocomputing, vol. 151, no. 2, pp. 737-744, 2015.

[24] X. Wan, L. Xu, H. Fang, and F. Yang, "Robust stability analysis for discrete-time genetic regulatory networks with probabilistic time delays," Neurocomputing, vol. 124, no. 2, pp. 72-80, 2014.

[25] Q. Ye and B. Cui, "Mean square exponential and robust stability of stochastic discrete-time genetic regulatory networks with uncertainties," Cognitive Neurodynamics, vol. 4, no. 2, pp. 165$176,2010$.

[26] K. Mathiyalagan and R. Sakthivel, "Robust stabilization and $H_{\infty}$ control for discrete-time stochastic genetic regulatory networks with time delays," Canadian Journal of Physics, vol. 90, no. 10, pp. 939-953, 2012.

[27] J. Hu, Z. Wang, D. Chen, and F. E. Alsaadi, "Estimation, filtering and fusion for networked systems with networkinduced phenomena: new progress and prospects," Information Fusion, vol. 31, pp. 65-75, 2016.

[28] Y. Wang, J. Cao, and L. Li, "Global robust power-rate stability of delayed genetic regulatory networks with noise perturbations," Cognitive Neurodynamics, vol. 4, no. 1, pp. 81-90, 2010.

[29] J. Hu, Z. Wang, B. Shen, and H. Gao, "Quantised recursive filtering for a class of nonlinear systems with multiplicative noises and missing measurements," International Journal of Control, vol. 86, no. 4, pp. 650-663, 2013.

[30] Y. Li, Y. Zhu, N. Zeng, and M. Du, "Stability analysis of standard genetic regulatory networks with time-varying delays and stochastic perturbations," Neurocomputing, vol. 74, no. 17, pp. 3235-3241, 2011.

[31] J. Hu, Z. Wang, S. Liu, and H. Gao, "A variance-constrained approach to recursive state estimation for time-varying complex networks with missing measurements," Automatica, vol. 64, pp. 155-162, 2016.

[32] B.-S. Chen, Y.-T. Chang, and Y.-C. Wang, "Robust $H_{\infty^{-}}$ stabilization design in gene networks under stochastic molecular noises: fuzzy-interpolation approach," IEEE Transactions on Systems, Man, and Cybernetics Part B: Cybernetics, vol. 38, no. 1, pp. 25-42, 2008.

[33] Y. Wang, Z. Ma, J. Shen, Z. Liu, and L. Chen, "Periodic oscillation in delayed gene networks with SUM regulatory logic and small perturbations," Mathematical Biosciences, vol. 220, no. 1, pp. 34-44, 2009. 
[34] P. Park, J. W. Ko, and C. Jeong, "Reciprocally convex approach to stability of systems with time-varying delays," Automatica, vol. 47, no. 1, pp. 235-238, 2011.

[35] K. Mathiyalagan, R. Sakthivel, and S. M. Anthoni, "New robust passivity criteria for discrete-time genetic regulatory networks with Markovian jumping parameters," Canadian Journal of Physics, vol. 90, no. 2, pp. 107-118, 2012.

[36] Q. Li, B. Shen, Y. Liu, and F. E. Alsaadi, "Event-triggered $H_{\infty}$ state estimation for discrete-time stochastic genetic regulatory networks with Markovian jumping parameters and timevarying delays," Neurocomputing, vol. 174, pp. 912-920, 2016.

[37] J. Hu, Z. Wang, H. Gao, and L. K. Stergioulas, "Probabilityguaranteed $H_{\infty}$ finite-horizon filtering for a class of nonlinear time-varying systems with sensor saturations," Systems and Control Letters, vol. 61, no. 4, pp. 477-484, 2012.

[38] N. Hou, H. Dong, Z. Wang, W. Ren, and F. E. Alsaadi, "Nonfragile state estimation for discrete Markovian jumping neural networks," Neurocomputing, vol. 179, pp. 238-245, 2016.

[39] J. Hu, J. Liang, D. Chen, D. Ji, and J. Du, "A recursive approach to non-fragile filtering for networked systems with stochastic uncertainties and incomplete measurements," Journal of the Franklin Institute, vol. 352, no. 5, pp. 1946-1962, 2015.

[40] R. Sakthivel, K. Mathiyalagan, S. Lakshmanan, and J. H. Park, "Robust state estimation for discrete-time genetic regulatory networks with randomly occurring uncertainties," Nonlinear Dynamics, vol. 74, no. 4, pp. 1297-1315, 2013.

[41] J. Hu, S. Liu, D. Ji, and S. Li, "On co-design of filter and fault estimator against randomly occurring nonlinearities and randomly occurring deception attacks," International Journal of General Systems, vol. 45, no. 5, pp. 619-632, 2016.

[42] H. Dong, Z. Wang, S. X. Ding, and H. Gao, "Finite-horizon reliable control with randomly occurring uncertainties and nonlinearities subject to output quantization," Automatica, vol. 52, pp. 355-362, 2015.

[43] J. Hu, Z. Wang, and H. Gao, "Recursive filtering with random parameter matrices, multiple fading measurements and correlated noises," Automatica, vol. 49, no. 11, pp. 3440-3448, 2013.

[44] S. Liu, G. Wei, Y. Song, and Y. Liu, "Error-constrained reliable tracking control for discrete time-varying systems subject to quantization effects," Neurocomputing, vol. 174, pp. 897-905, 2016.

[45] J. Hu, Z. Wang, H. Gao, and L. K. Stergioulas, "Extended Kalman filtering with stochastic nonlinearities and multiple missing measurements," Automatica, vol. 48, no. 9, pp. 20072015, 2012.

[46] G. Wei, S. Liu, Y. Song, and Y. Liu, "Probability-guaranteed set-membership filtering for systems with incomplete measurements," Automatica, vol. 60, pp. 12-16, 2015. 


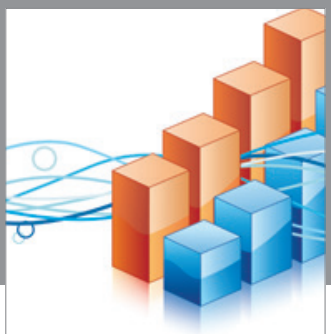

Advances in

Operations Research

vatem alat4

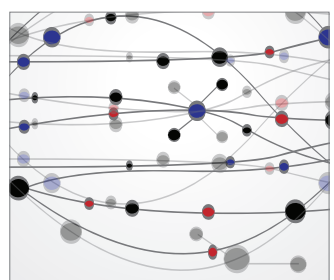

\section{The Scientific} World Journal
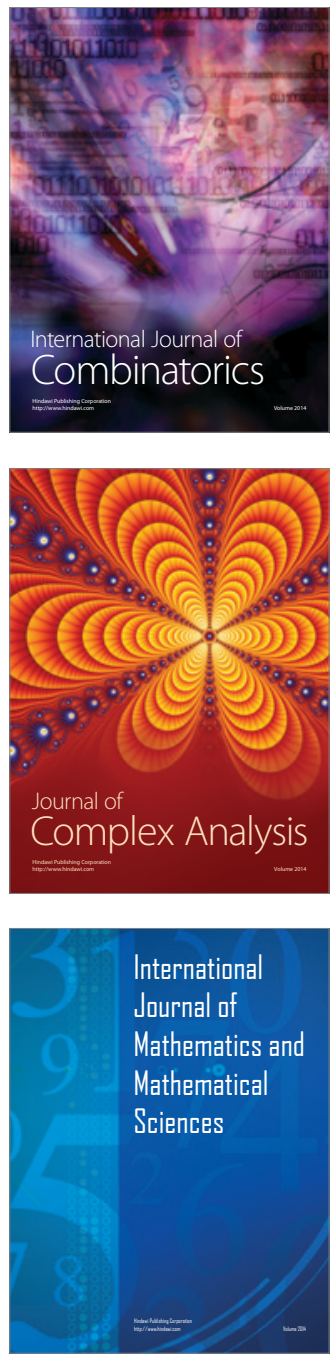
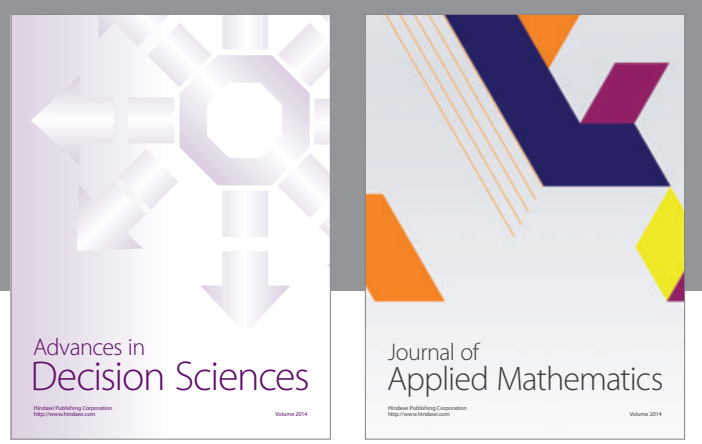

Algebra

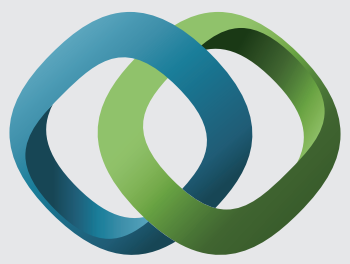

\section{Hindawi}

Submit your manuscripts at

http://www.hindawi.com
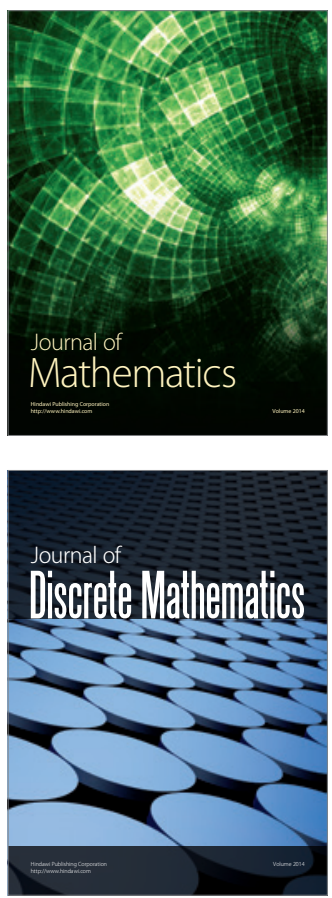

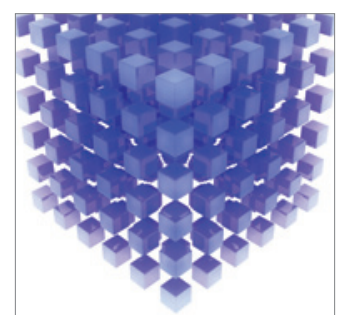

Mathematical Problems in Engineering
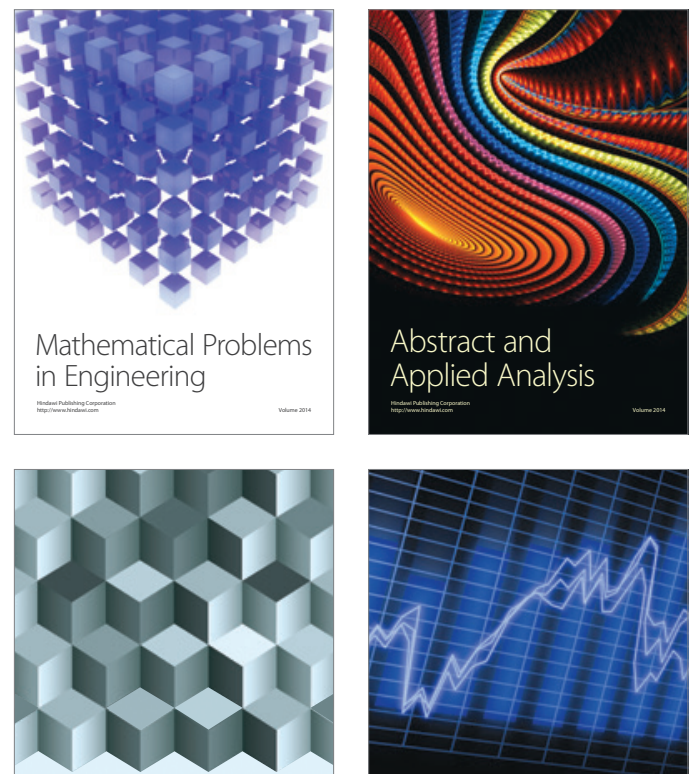

Journal of

Function Spaces

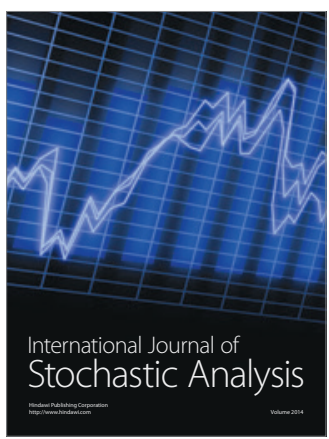

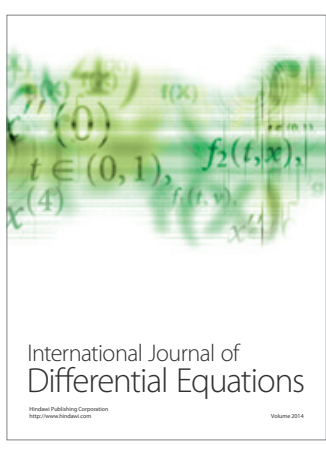
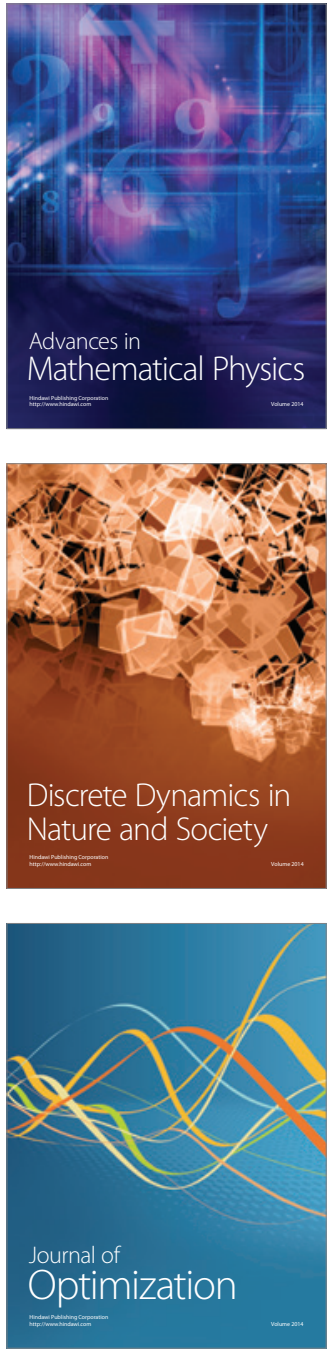